\title{
Hemoptysis as a rare presentation of cor triatriatum sinister
}

\author{
Sameh M. Said, MD, ${ }^{a}$ Ali N. Ibrahimiye, MD,${ }^{b}$ Rajesh Punn, MD,${ }^{c}$ and Frank L. Hanley, MD, \\ Rochester, Minn, and Palo Alto, Calif
}

From the ${ }^{\mathrm{a} D i v i s i o n}$ of Cardiovascular Surgery, Mayo Clinic, Rochester, Minn; ${ }^{\mathrm{b}}$ Division of Pediatric Cardiac Sur-
gery and ${ }^{\mathrm{c} D i v i s i o n}$ of Pediatric Cardiology, Stanford University, Palo Alto, Calif.
Disclosures: Authors have nothing to disclose with regard to commercial support.
Received for publication July 13, 2015; accepted for publication July $29,2015$.
Address for reprints: Sameh M. Said, MD, Division of Cardiovascular Surgery, Mayo Clinic, 200 First St SW,
Rochester, MN 55905 (E-mail: smsaid75@ yahoo.com).
J Thorac Cardiovasc Surg 2015;150:e73-5.
0022-5223/\$36.00
Copyright @ 2015 by The American Association for Thoracic Surgery
http://dx.doi.org/10.1016/j.jtcvs.2015.07.095

Cor triatriatum sinister is a rare cardiac malformation in which the left atrium is divided into 2 chambers by an obstructing membrane, a proximal chamber that receives the pulmonary venous drainage and a distal chamber that contains the mitral valve and left atrial appendage. The clinical presentation and severity of symptoms depend on the degree of obstruction and the presence or absence of atrial level shunting.

Most cases are detected early in life. We report a 16-yearold male who presented with intermittent hemoptysis and underwent extensive evaluation that identified a cor triatriatum sinister.

\section{CASE REPORT}

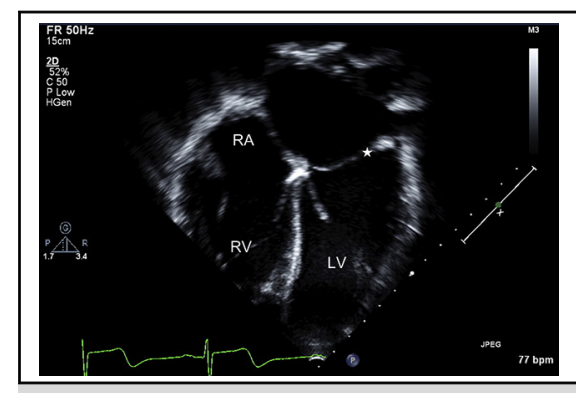

Echocardiography of the nearly complete septum above the mitral valve.

\section{Central Message}

Unusual etiology of hemoptysis due to cor triatriatum sinister.

See Editorial Commentary page e77.

A 16-year-old male with history of reactive airway disease presented with hemoptysis. He was evaluated
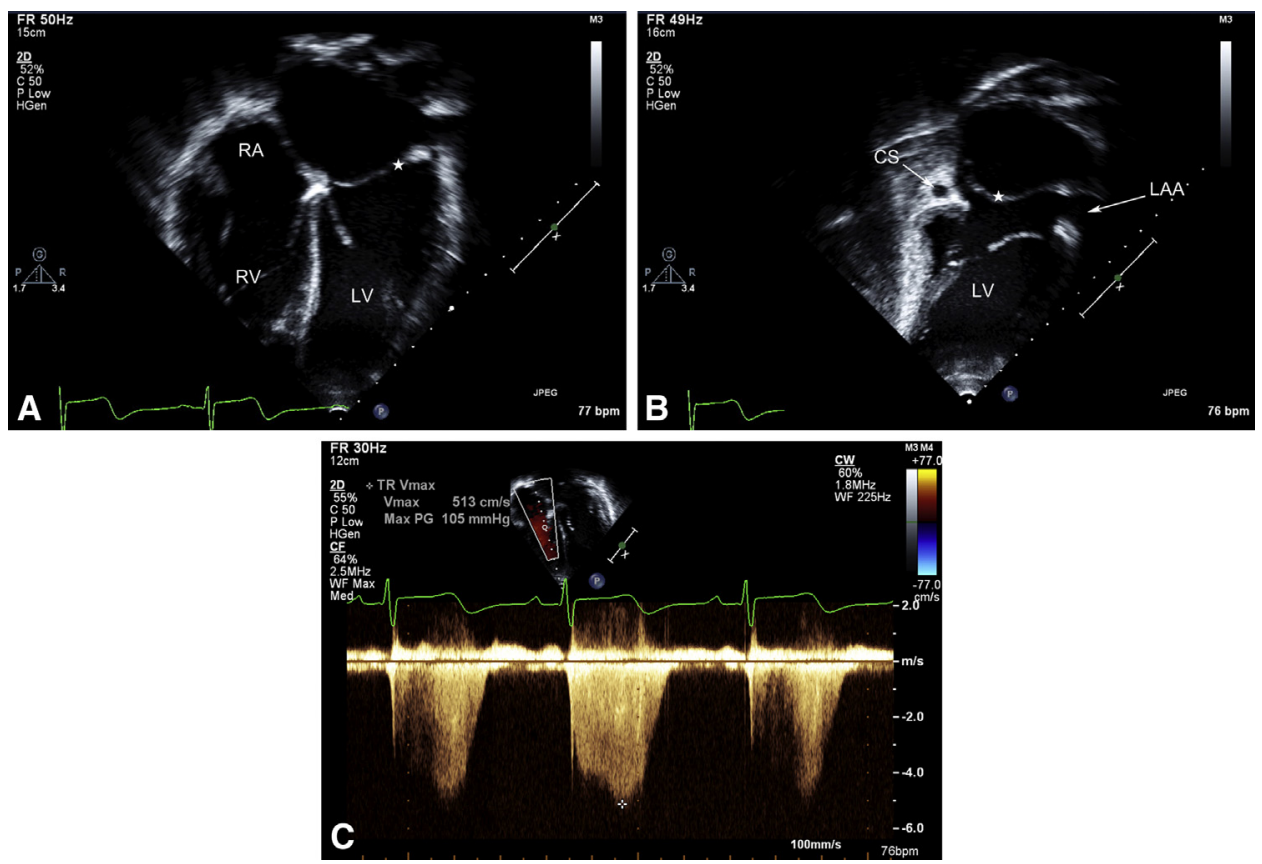

FIGURE 1. A, Transthoracic echocardiogram images showing an apical 4-chamber view with the nearly complete septum ( $\star$ ) above the left ventricle and mitral valve. B, Apical 2-chamber view showing the membrane $(\star)$ superior to the left atrial appendage and coronary sinus. C, The tricuspid valve regurgitation jet estimates a right ventricular pressure of $>100 \mathrm{~mm} \mathrm{Hg}$ above the right atrial pressure by continuous-wave Doppler. $R A$, Right atrium; $R V$, right ventricle; $L V$, left ventricle; $C S$, coronary sinus; $L A A$, left atrial appendage; $T R V_{\max }$, tricuspid valve regurgitation maximum velocity. 


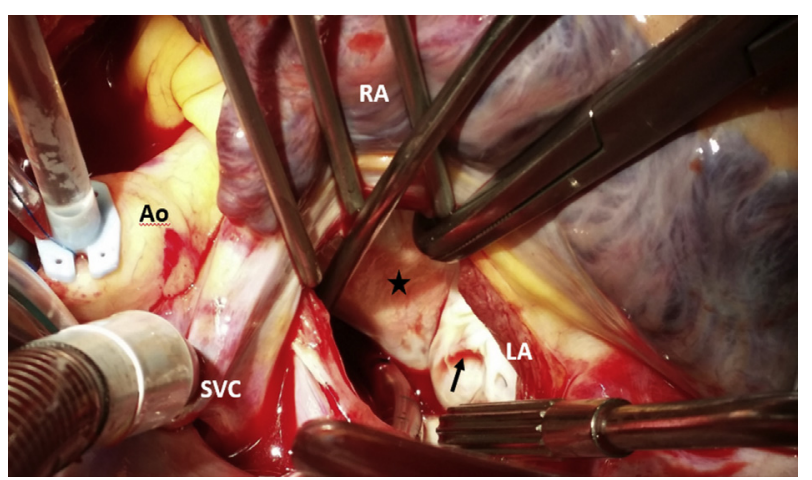

FIGURE 2. Intraoperative photo showing the nearly complete septum ( $\star$ ) through the opened left atrium. The black arrow points to the communication between the 2 chambers of the left atrium. RA, Right atrium; Ao, aorta; $S V C$, superior vena cava; $L A$, left atrium.

extensively to rule out infectious or hematologic etiologies, and his hemoptysis was thought to be secondary to chronic inflammation. He had been experiencing attacks of hemoptysis once a month for a little more than 1 year, with bright-red bleeding slightly greater than 1 teaspoon. He had no history of fever, chest pain, or other bleeding diatheses. Initial evaluation included bronchoscopy and computed tomography (CT) scan, which revealed possible pulmonary fibrosis. The patient was transferred to our center for further treatment of hypersensitivity pneumonitis and pulmonary fibrosis.

After admission, review of the CT scan revealed pulmonary artery enlargement and right ventricular hypertrophy. Transthoracic echocardiography showed an obstructing membrane in the left atrium (cor triatriatum sinister) (Figure 1, A) superior to the left atrial appendage (Figure $1, B$ ), with a mean gradient of $20 \mathrm{mmHg}$ across it. The estimated right ventricular pressure was $>100 \mathrm{mmHg}$ (Figure 1,C). The interventricular septum appeared flattened, and the right ventricle was moderately depressed; however left ventricular systolic function was preserved.

The patient was taken to the operating room and through a standard left atrial approach, an obstructing fibromuscular membrane was identified (Figure 2) with 2 small openings (Figure $3, A$ and $B$ ) about 3 to $5 \mathrm{~mm}$ each, one near the aortic root and the other near the crux of the heart. There was no atrial septal defect (Lam class A); however, there was a nearly complete septum of cor triatriatum, and through 1 of these small communications, access to the inferior chamber was secured, and the membrane was circumferentially and completely resected (Figure 4, $A$ and $B$ ).

\section{COMMENT}

First described by Church in 1868 , cor triatriatum sinister represents $0.1 \%$ of all congenital heart defects in which the left atrium is divided into 2 chambers. ${ }^{1}$ The proximal chamber contains all pulmonary veins, and the distal chamber contains the mitral valve and the LAA. It can occur as an isolated anomaly (classic) or with other cardiac defects (atypical). The position of the LAA is important for differentiating cor triatriatum from the supramitral ring in which the membrane is below the LAA. The embryologic basis is unclear, but theories of malseptation, malincorporation, or entrapment have been proposed. $^{2}$

Cor triatriatum sinister is a rare but surgically correctable anomaly, with the first surgical repair reported by Lewis and colleagues ${ }^{3}$ in 1956 using inflow occlusion and hypothermia to incise the obstructing diaphragm. Vineberg ${ }^{4}$ reported using a finger fracture technique through the LAA.

A wide spectrum of presentations may be encountered based on the degree of obstruction and the presence or absence of interatrial communication. Affected infants may present with shock, pulmonary edema, and respiratory failure.

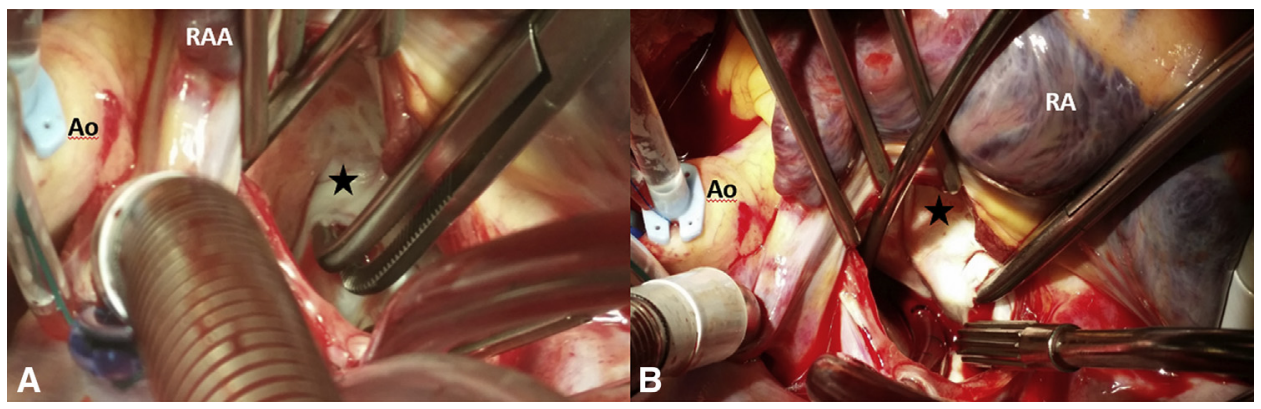

FIGURE 3. Intraoperative photos showing the septum $(\star)$ through the opened left atrium. A, A right angled-clamp passing through the communication between the 2 chambers. B, The right angled-clamp in the lower chamber, which contains the mitral valve and the left atrial appendage. $R A A$, Right atrial appendage; $A o$, aorta; $R A$, right atrium. 


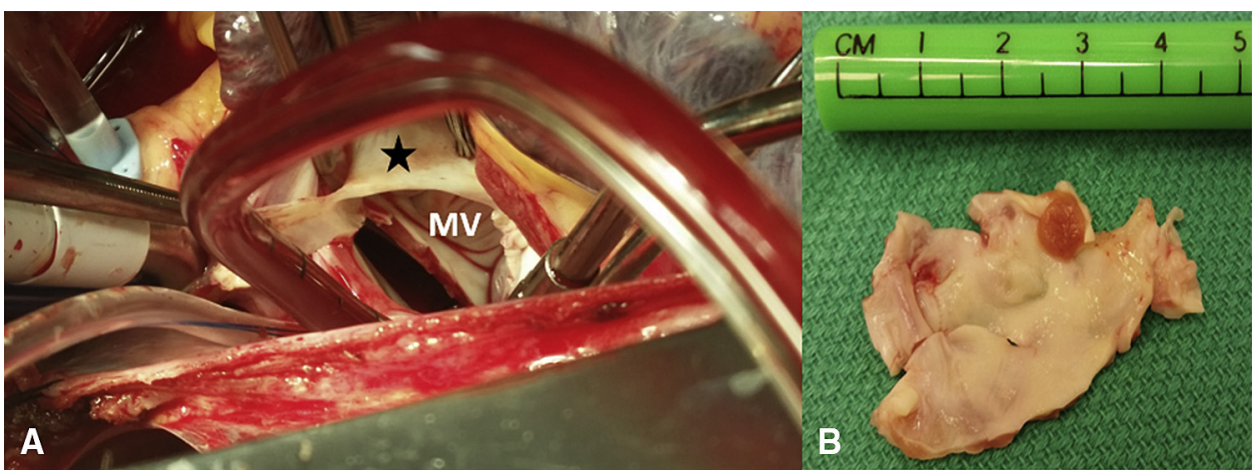

FIGURE 4. Intraoperative photos showing A, the resected septum ( $\star$ ), with the mitral valve is shown in the lower chamber beneath the septum, and B, the completely resected septum. $M V$, Mitral valve.

Saxena and coworkers ${ }^{5}$ reported their experience with 25 patients who underwent surgical correction of cor triatriatum. The mean age was 27 years, and all patients underwent membrane resection using cardiopulmonary bypass. Twenty patients ( $80 \%$ ) underwent concomitant cardiac procedures. The most common type was Lam class A1 (atrial septal defect between the proximal chamber and the right atrium), followed by Lam class A (no atrial septal defect). The most common associated anomaly was atrial septal defect $(28 \%)$, followed by partial anomalous pulmonary venous drainage $(12 \%)$. There was no early mortality in this series, and 10 -year survival was $83 \%$, with all patients in New York Heart Association class I or II at a mean follow-up of 12.8 years.

In another report from Alphonso and coworkers, ${ }^{6} 28$ patients with cor triatriatum underwent resection of the membrane. Fifteen of the patients were under age 1 year, and the median age at presentation was 6 months. The main presenting symptoms were tachypnea, failure to thrive, shock, cyanosis, and exertional dyspnea. Six patients had severe symptoms and underwent emergency surgery. There was 1 early death, and the median follow-up interval was 98 months.

Other atypical presentations of cor triatriatum include atrial fibrillation, ${ }^{7}$ fatigue, ${ }^{8}$ and cryptogenic stroke. ${ }^{9}$ Our patient presented with recurrent hemoptysis secondary to severe pulmonary venous hypertension, which is unusual but can be explained by the nearly complete obstruction of pulmonary venous drainage owing to the presence of the membrane.

Despite rare cases of successful balloon catheter dilation of the obstructing membrane, ${ }^{10}$ surgical resection is the main treatment of choice, with excellent results and no recurrence.

\section{References}

1. Lam CR, Green E, Drake E. Diagnosis and surgical correction of 2 types of triatrial heart. Surgery. 1962;51:127-37.

2. Van Praagh R, Corsini I. Cor triatriatum: pathologic anatomy and a consideration of morphogenesis based on 13 postmortem cases and a study of normal development of the pulmonary vein and atrial septum in 83 human embryos. Am Heart J. 1969; 78:379-405.

3. Lewis FJ, Varco RL, Taufic M, Niazi SA. Direct vision repair of triatrial heart and total anomalous pulmonary venous drainage. Surg Gynecol Obstet. 1956;102:713-20.

4. Vineberg A, Gialloreto O. Report of a successful operation for stenosis of common pulmonary vein (cor triatriatum). Can Med Assoc J. 1956;74:719-23.

5. Saxena P, Burkhart HM, Schaff HV, Daly R, Joyce LD, Dearani JA. Surgical repair of cor triatriatum sinister: the Mayo Clinic 50-year experience. Ann Thorac Surg. 2014;97:1659-63.

6. Alphonso N, Nørgaard MA, Newcomb A, d'Udekem Y, Brizard CP, Cochrane A. Cor triatriatum: presentation, diagnosis, and long-term surgical results. Ann Thorac Surg. 2005;80:1666-71.

7. Zepeda IA, Morcos P, Castellanos LR. Cor triatriatum sinister identified after new onset atrial fibrillation in an elderly man. Case Rep Med. 2014;2014:674018.

8. Strickland PT, Pernetz MA, Jokhadar M, Hartlage G, Clements S. Cor triatriatum sinister: a patient, a review, and some unique findings. Echocardiography. 2014; 31:790-4.

9. Ridjab DA, Wittchen F, Tschishow W, Buddecke J, Lamp B, Manegold J, et al. Cor triatriatum sinister and cryptogenic stroke. Herz. 2015;40:447-8.

10. Kerkar P, Vora A, Kulkarni H, Narula D, Goyal V, Dalvi B. Percutaneous balloon dilatation of cor triatriatum sinister. Am Heart J. 1996;132:888-91. 